\title{
Steroid avoidance in pediatric transplant patients is safe
}

Steroid avoidance is not associated with an increased risk of clinical rejection in pediatric kidney transplant recipients, show investigators of a randomized, prospective, multicenter trial. "The design of this trial was based on very exciting single-center data where we used extended daclizumab to provide additional immunosuppression in the first 6 months after transplantation while completely eliminating steroid use", says researcher Minnie Sarwal. "This approach was a departure from usual care in children where steroid withdrawal was being practiced either very selectively, or not at all."

The promising outcomes of this pilot study led to the enrollment of 130 pediatric and adolescent kidney transplant patients (aged 0-21 years) who were randomly assigned to steroid-free or steroid-based immunosuppression. Renal allograft biopsies were planned at the time of transplantation and at 6,12 and 24 months after transplantation to assess clinical and subclinical determinants of the evolution of chronic histological renal allograft injury.

Sarwal et al. found that the primary efficacy end point of the difference in linear growth between the two groups at 1 year was not significant. However, when growth was stratified for age, the growth rate for children aged $<5$ years was significantly higher in the steroid-free than in the steroid-based group. BMI, hospitalization rates and infection rates at 3 years post-transplantation did not differ between the two groups.

\section{4 ...the growth rate for children aged $<5$ years was significantly higher in the steroid-free than in the steroid-based group 77}

A histological substudy of the trial, outlined in a paper by Naesens et al., found no significant difference between the two groups in the primary safety end point of biopsy-proven acute rejection at 12 months. However, there was a higher incidence trend of recurrent acute rejection in the steroid-based group, and antibody-mediated rejection in the steroid-free group, findings that might have clinical importance for patients with immunological higher risk profile. Chronic histological damage significantly increased over time, but there was no difference in the prevalence or evolution of histological lesions or calculated chronic histological damage scores between the two groups. Graft function and survival were similar in both groups at all time points. Small recipient size and higher donor age were independent risk factors for progressive chronic tubulointerstitial damage of renal allografts.

"This study has revolutionized the care for many children by letting their physicians safely treat them without steroids and their crippling adverse effects", says Sarwal. "We have now developed a blood gene-based test to diagnose and predict transplant rejection without the need of a biopsy, providing the first means to monitor for acute rejection by a blood sample and avoid the morbidity and cost of a biopsy."

\section{Helene Myrvang}

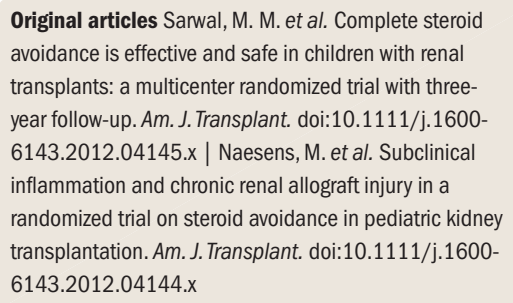

